\title{
Higher accurate approximate solutions for the simple pendulum in terms of elementary functions
}

\author{
Augusto Beléndez $^{1,2}$, Jorge Francés ${ }^{1}$, Manuel Ortuño ${ }^{1,2}$, Sergi Gallego ${ }^{1,2}$ \\ and José Guillermo Bernabeu ${ }^{1,2}$
}

(1) Departamento de Física, Ingeniería de Sistemas y Teoría de la Señal Universidad de Alicante. Apartado 99. E-03080 Alicante. SPAIN

(2) Instituto Universitario de Física Aplicada a las Ciencias y las Tecnologías Universidad de Alicante. Apartado 99. E-03080 Alicante. SPAIN

E-mail: a.belendez@ua.es

Corresponding author: Augusto Beléndez

Phone: +34-96-5903651

Fax: +34-96-5909750 


\begin{abstract}
A closed-form approximate expression for the solution of a simple pendulum in terms of elementary functions is obtained. To do this, the exact expression for the maximum tension of the string of the pendulum is first considered and a trial approximate solution depending of some parameters is used and which is substituted in the tension equation. We obtain the parameters for the approximate by means of a term-by-term comparison of the power series expansion for the approximate maximum tension with the corresponding series for the exact one. We believe that the present letter may be a suitable and fruitful exercise for teaching and better understanding nonlinear oscillations of a simple pendulum in undergraduate courses on classical mechanics.
\end{abstract}

KEY WORDS: Nonlinear pendulum; Tension; Approximate solutions. 
The simple pendulum is one of the most popular examples analysed in textbooks and undergraduate courses in physics [1] and it is perhaps the most investigated oscillatory motion in physics. Many nonlinear phenomena in real world are governed by pendulumlike differential equations [2]. The nonlinear differential equation for the simple pendulum can be exactly solved and the period and periodic solution expressions involve the complete elliptic integral of the first kind and the Jacobi elliptic functions, respectively. Due to this, several approximation schemes have been developed to investigate the situation for large amplitude oscillations of a simple pendulum, and several approximations for its large-angle period have been suggested (a summary of most of them can be found in [3-5]). However, it is not as easy to obtain an accurate approximate solution in terms of elementary functions. The most common approximation is to consider a trigonometric approximate solution in the form $\theta(t) \approx \theta_{0} \cos \left(\omega_{a} t\right)$ [6], where $\omega_{a}$ is one of the suitable approximations for the exact frequency and it is a function of the angular amplitude $\theta_{0}$. In this letter we obtain some analytical approximate solutions for the simple pendulum in terms only of elementary functions (polynomial and trigonometric functions). To do this, we don't use the nonlinear differential equation of motion for the simple pendulum but the exact expression of the tension in the pendulum string at arbitrary angular position and the maximum value for this tension. Our results allows us not only to obtain an approximate expression for the solution but also a trigonometric approximation for the tension in the string whose maximum relative error is as low as $0.27 \%$ for amplitudes less than $\pi / 2$ rad. We believe this study, or parts of it, can be useful not only in advanced but also in introductory university courses of classical mechanics in a second or a third year course. In a recent letter [6], Lima obtained a trigonometric approximation for the tension in the string of a simple pendulum accurate for all amplitudes. To obtain this formula, Lima takes into account the expression for the tension $\mathrm{T}$ in the pendulum string at an arbitrary angular position $\theta(t)$, which is given as follows

$$
\mathrm{T}=m g \cos \theta+m l\left(\frac{d \theta}{d t}\right)^{2}
$$


where $m$ is the mass of the bob, $l$ is the length of the string and $g$ is the acceleration of gravity, and the formulae for the exact minimum and maximum values of the tension have the following expressions

$$
\begin{gathered}
\mathrm{T}_{\text {min }}=m g \cos \theta_{0} \\
\mathrm{~T}_{\text {max }}=m g\left(3-2 \cos \theta_{0}\right)
\end{gathered}
$$

where $\theta_{0}$ is the angular amplitude. For deriving a trigonometric approximation for the tension valid for all amplitudes Lima considers the following approximate solution to the pendulum equation of motion [6]

$$
\theta(t) \approx \theta_{0} \cos \left(\omega_{a} t\right)
$$

where $\omega_{a}$ is some suitable approximation for the exact pendulum frequency $\omega$. In particular, Lima takes into account Ganley's formula for the approximate frequency [7]. However, the results Lima obtained cannot give a correct value for the maximum tension for all amplitudes. For instance, for $\theta_{0}=\pi / 4$ and $\pi / 2$, the approximate maximum tensions obtained by Lima are 1.5704 and 2.7738, respectively. However, it is easy to verify from Eq. (3) that the values for the exact maximum tension are 1.5858 and 3 , respectively. Evidently, these differences are due to the election of Eq. (4) for the solution.

Lima's letter gave us the idea of using the exact expressions for the tension (Eq. (1)) and the maximum tension (Eq. (3)) to obtain an approximate expression for the solution of the pendulum equation of motion in term of elementary functions. In this sense, we are going to do an inverse procedure that those used by Lima.

The exact solution for the simple pendulum is periodic and then it can be represented as a Fourier series

$$
\theta(t)=\sum_{n=0}^{\infty} c_{2 n+1}\left(\theta_{0}\right) \cos [(2 n+1) \omega t]
$$


where $\omega$ is the exact frequency, the Fourier coefficients $c_{2 n+1}$ depend on the amplitude $\theta_{0}$ and we have considered that $\theta(0)=\theta_{0}$ and $\left(\frac{d \theta}{d t}\right)_{t=0}=0$ as initial conditions. Now we approximate the periodic solution in Eq. (6) by a trigonometric polynomial as follows (only the first two terms in Eq. (5))

$$
\theta(t) \approx b_{1}\left(\theta_{0}\right) \cos \left(\omega_{a} t\right)+b_{3}\left(\theta_{0}\right) \cos \left(3 \omega_{a} t\right)
$$

where $\omega_{a}$ is some suitable approximation for the exact frequency $\omega$, and $b_{1}$ and $b_{3}$ are two coefficients which have to be obtained. As $\theta(0)=\theta_{0}$ we can write Eq. (6) as follows

$$
\theta(t) \approx\left[\theta_{0}-b_{3}\left(\theta_{0}\right)\right] \cos \left(\omega_{a} t\right)+b_{3}\left(\theta_{0}\right) \cos \left(3 \omega_{a} t\right)
$$

and only one coefficient has to be determined. For $\omega_{a}$ we use the Carvalhaes and Suppes' expression obtained using the arithmetic-geometric mean $[8,9]$ which can be written as follows

$$
\omega_{a}=\frac{1}{4} \sqrt{\frac{g}{l}}\left(1+\sqrt{\cos \left(\frac{\theta_{0}}{2}\right)}\right)^{2}
$$

The error for Eq. (8) is lower than $1 \%$ for $\theta_{0} \leq 163^{\circ}$ [5]. Now we expand the maximum tension in Eq. (3) in a Maclaurin series, and we obtain

$$
\mathrm{T}_{\max } \approx m g\left(1+\theta_{0}^{2}-\frac{1}{12} \theta_{0}^{4}+\frac{1}{360} \theta_{0}^{6}-\ldots\right)
$$

and we also approximate the coefficient $b_{3}$ as follows 
Higher accurate approximate solutions for the simple pendulum in terms of elementary functions Augusto Beléndez, Jorge Francés, Manuel Ortuño, Sergi Gallego and José Guillermo Bernabeu, European Journal of Physics, Vol. 31, $\mathrm{N}^{\mathrm{o}}$ 3, L65-L70 (2010) doi: 10.1088/0143-0807/31/3/L04

$$
b_{3}\left(\theta_{0}\right)=a_{3} \theta_{0}^{3}
$$

Substituting Eqs. (7), (8) and (10) into Eq. (1) we can easily obtain the following approximate expression for the maximum tension, $\mathrm{T}_{\max }^{a p p}$,

$$
\mathrm{T}_{\max }^{a p p}=m g\left[1+\frac{1}{16} \theta_{0}^{2}\left(1-4 a_{3} \theta_{0}^{2}\right)^{2}\left(1+\sqrt{\cos \left(\frac{\theta_{0}}{2}\right)}\right)^{4}\right]
$$

The power-series expansion for this approximate maximum tension is given as follows

$$
\mathrm{T}_{\max }^{a p p} \approx m g\left[1+\theta_{0}^{2}-\left(\frac{1}{8}+8 a_{3}\right) \theta_{0}^{4}+\ldots\right]
$$

Comparing the first terms in the series expansions of Eqs. (9) and (12) we obtain

$$
a_{3}=-\frac{1}{192}
$$

and the approximate maximum tension can be written as follows

$$
\mathrm{T}_{\text {max }}^{a p p}=m g\left[1+\frac{1}{16} \theta_{0}^{2}\left(1+\frac{1}{48} \theta_{0}^{2}\right)^{2}\left(1+\sqrt{\cos \left(\frac{\theta_{0}}{2}\right)}\right)^{4}\right]
$$

whose takes the values 1.5851 and 2.9578 for $\theta_{0}=\pi / 4$ and $\pi / 2 \mathrm{rad}$, respectively (from Eq. (3) it is easy to verify that the exact values are 1.5858 and 3, respectively). The approximate solution for the pendulum can be then written as follows 


$$
\theta(t) \approx\left(\theta_{0}+\frac{1}{192} \theta_{0}^{3}\right) \cos \left(\omega_{a} t\right)-\frac{1}{192} \theta_{0}^{3} \cos \left(3 \omega_{a} t\right)
$$

A better approximation can be obtained if we consider the following approximation for the coefficient $b_{3}$

$$
b_{3}\left(\theta_{0}\right)=a_{3} \theta_{0}^{3}+a_{5} \theta_{0}^{5}
$$

Substituting Eqs. (7), (8) and (16) into Eq. (1), calculating the approximate maximum tension, expanding this maximum tension in power-series and comparing the first terms of this expansion and the expansion in Eq. (9) we obtain

$$
a_{3}=-\frac{1}{192} \text { and } a_{5}=-\frac{23}{61440}
$$

and the approximate maximum tension can be written as follows

$$
\mathrm{T}_{\max }^{a p p}=m g\left[1+\frac{1}{16} \theta_{0}^{2}\left(1+\frac{1}{48} \theta_{0}^{2}+\frac{23}{15360} \theta_{0}^{4}\right)^{2}\left(1+\sqrt{\cos \left(\frac{\theta_{0}}{2}\right)}\right)^{4}\right]
$$

Now the values for the approximate maximum tension for $\theta_{0}=\pi / 4$ and $\pi / 2 \mathrm{rad}$ are 1.5858 and 2.9919, respectively (the exact values are 1.5858 and 3, respectively). This implies that the maximum relative error for the approximate maximum tension given in Eq. (18) is less than $0.27 \%$ for $\theta_{0}<\pi / 2 \mathrm{rad}$. The relative error obtained by Lima for the same angle was 7\% [6]. In Figure 1 we have plotted the maximum tension in the string of a simple pendulum, in units of the weight $m g$, as a function of the angular amplitude. In this figure we have included the exact expression as well as Lima's formula (Eq. (18) in ref. [6]) and Eq. (18) in this paper. As we can see, the approximate formula for the maximum tension given in this letter, found in (18), yields values much closer to the exact ones, obtained using Eq. (3), than those yields by the Lima's formula. 
The approximate solution for the pendulum are given as follows

$$
\theta(t)=\left(\theta_{0}+\frac{1}{192} \theta_{0}^{3}+\frac{23}{61440} \theta_{0}^{5}\right) \cos \left(\omega_{a} t\right)-\left(\frac{1}{192} \theta_{0}^{3}+\frac{23}{61440} \theta_{0}^{5}\right) \cos \left(3 \omega_{a} t\right)
$$

The approximate periodic solution, $\theta(t) / \theta_{0}$ in (18), and the exact one [10], are plotted in Figure 2 for $\theta_{0}=2 \pi / 3 \mathrm{rad}$. In this figure, parameter $h$ is defined as $h=\omega_{e x} t / 2 \pi$. This figure shows that Eqs. (8) and (18) can provide high accurate approximations to the exact frequency and the exact periodic solutions for $\theta_{0}<2 \pi / 3 \mathrm{rad}$.

Now we compare the Fourier series expansion of the exact solution [11] with the approximate solution in Eq. (18) for different values of the amplitude. For $\theta_{0}=\pi / 6 \mathrm{rad}$ we obtain

$$
\begin{aligned}
& \frac{\theta_{\mathrm{ex}}(t)}{\theta_{0}}=1.00145 \cos \omega_{\mathrm{ex}} t-0.00145284 \cos 3 \omega_{\mathrm{ex}} t+0.00000377745 \cos 5 \omega_{\mathrm{ex}} t-\ldots \\
& \frac{\theta_{a}(t)}{\theta_{0}}=1.00146 \cos \omega_{a} t-0.00145603 \cos 3 \omega_{a} t
\end{aligned}
$$

For $\theta_{0}=\pi / 3 \mathrm{rad}$ we obtain

$$
\begin{aligned}
& \frac{\theta_{\mathrm{ex}}(t)}{\theta_{0}}=1.00607 \cos \omega_{\mathrm{ex}} t-0.00613545 \cos 3 \omega_{\mathrm{ex}} t+0.0000661616 \cos 5 \omega_{\mathrm{ex}} t-\ldots \\
& \frac{\theta_{a}(t)}{\theta_{0}}=1.00616 \cos \omega_{a} t-0.006161763 \cos 3 \omega_{a} t
\end{aligned}
$$

For $\theta_{0}=\pi / 2 \operatorname{rad}$ we obtain

$$
\frac{\theta_{\mathrm{ex}}(t)}{\theta_{0}}=1.01487 \cos \omega_{\mathrm{ex}} t-0.0152493 \cos 3 \omega_{\mathrm{ex}} t+0.00039542 \cos 5 \omega_{\mathrm{ex}} t-\ldots
$$


$\frac{\theta_{a}(t)}{\theta_{0}}=1.01513 \cos \omega_{a} t-0.0115130 \cos 3 \omega_{a} t$

These equations allow us to compare the first Fourier coefficients of the Fourier series expansions of exact and analytical approximate solutions for different values of $\theta_{0}$.

In summary, in this letter we have made use of Carvalhaes and Suppes approximate formula for the pendulum period for deriving a simple and accurate solution for the pendulum equation of motion in terms of elementary functions. To do this we use the expression for the maximum tension in the pendulum string. We have also obtained a trigonometric approximation for the tension in the string whose maximum error is less than $0.27 \%$ for all values of the amplitude less than $\pi / 2 \mathrm{rad}$, which is much better than the value of $7 \%$ obtained by Lima. The study considered in this letter reveals the accuracy of the approximate expressions for the period and the periodic solution, which can have pedagogical interest for undergraduate physics students. Finally, following the schema used in this letter it would be possible to obtain more accurate solution considering more trigonometric terms in Eq. (7). The following approximation would be $\theta(t) \approx\left[\theta_{0}-b_{3}\left(\theta_{0}\right)-b_{5}\left(\theta_{0}\right)\right] \cos \left(\omega_{a} t\right)+b_{3}\left(\theta_{0}\right) \cos \left(3 \omega_{a} t\right)+b_{5}\left(\theta_{0}\right) \cos \left(5 \omega_{a} t\right)$.

\section{Acknowledgments}

This work was supported by the "Vicerrectorado de Tecnología e Innovación Educativa" of the University of Alicante, Spain (GITE-09006-UA). 


\section{References}

[1] Baker G L and Blackburn J A 2005 The Pendulum: A Case Study in Physics (Oxford University Press: Oxford)

[2] Lima F M S 2008 Simple 'log formulae' for the pendulum motion valid for any amplitude Eur. J. Phys. 29 1091-8

[3] Beléndez A, Rodes JJ, Beléndez T and Hernández A 2009 Approximation for a large-angle simple pendulum period Eur. J. Phys. 30 L25-8

[4] Qing-Xin Y and Pei D 2009 Comment on 'Approximation for a large-angle simple pendulum period' Eur. J. Phys. 30 L79-82

[5] Beléndez A, Rodes JJ, Beléndez T and Hernández A 2009 Reply to "Comments on Approximation for a large-angle simple pendulum period" Eur. J. Phys. 30 L83-86

[6] Lima F M S 2009 A trigonometric approximation for the tension in the string of a simple pendulum accurate for all amplitudes Eur. J. Phys. 30 L95-102

[7] Ganley W P 1985 Simple pendulum approximation Am. J. Phys. 53 73-6

[8] Carvalhaes C G and Suppes P 2008 Approximation for the period of the simple pendulum based on the arithmetic-geometric mean Am. J. Phys 76 1150-4

[9] Carvalhaes C G and Suppes P 2009 The high-precision computation of the period of the simple pendulum Rev. Bras. Ens. Phys 31 art2701

[10] Beléndez A, Pascual C, Méndez DI, Beléndez T and Neipp C 2007 Exact solution for the nonlinear pendulum Rev. Bras. Ens. Phys 29 645-8

[11] Gimeno E and Beléndez A 2009 Rational-harmonic balancing approach to nonlinear phenomena governed by pendulum-like differential equations $Z$. Naturforsch. 63a 131-139 


\section{FIGURE CAPTIONS}

Figure 1.- Maximum tension in the string of a simple pendulum, in units of the weight $m g$, as a function of the angular amplitude. In this figure we have included the exact expression as well as Lima's formula (Eq. (18) in ref. [6]) and Eq. (18) in this letter.

Figure 2.- Comparison of the normalized approximate solution in Eq. (18) ( $\triangle$ and dashed line) with the exact solution ( $O$ and continuous line) for $\theta_{0}=2 \pi / 3$ rad. 
FIGURE 1

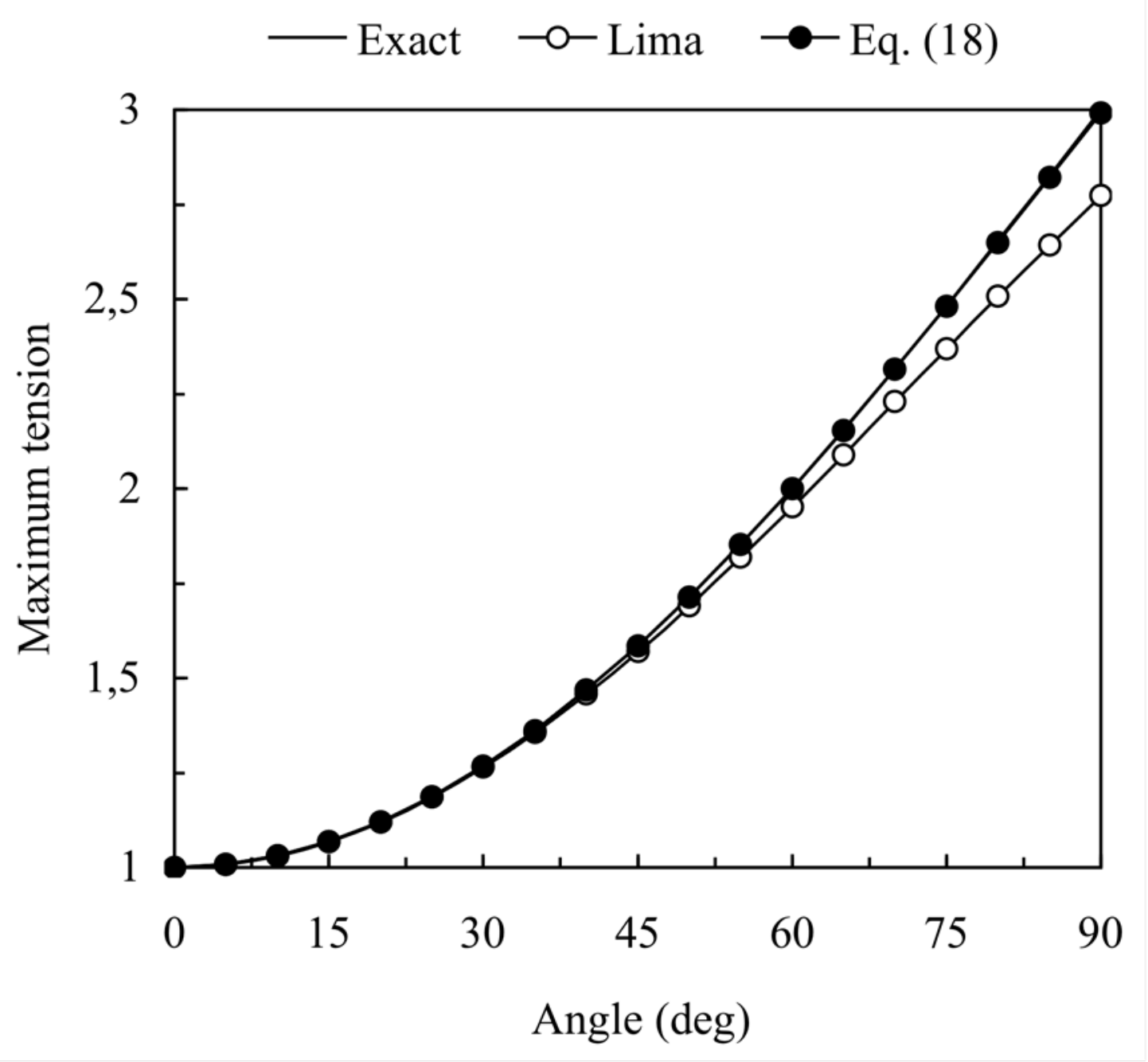


Higher accurate approximate solutions for the simple pendulum in terms of elementary functions Augusto Beléndez, Jorge Francés, Manuel Ortuño, Sergi Gallego and José Guillermo Bernabeu, European Journal of Physics, Vol. 31, No 3, L65-L70 (2010) doi: $\underline{10.1088 / 0143-0807 / 31 / 3 / L 04}$

\section{FIGURE 2}

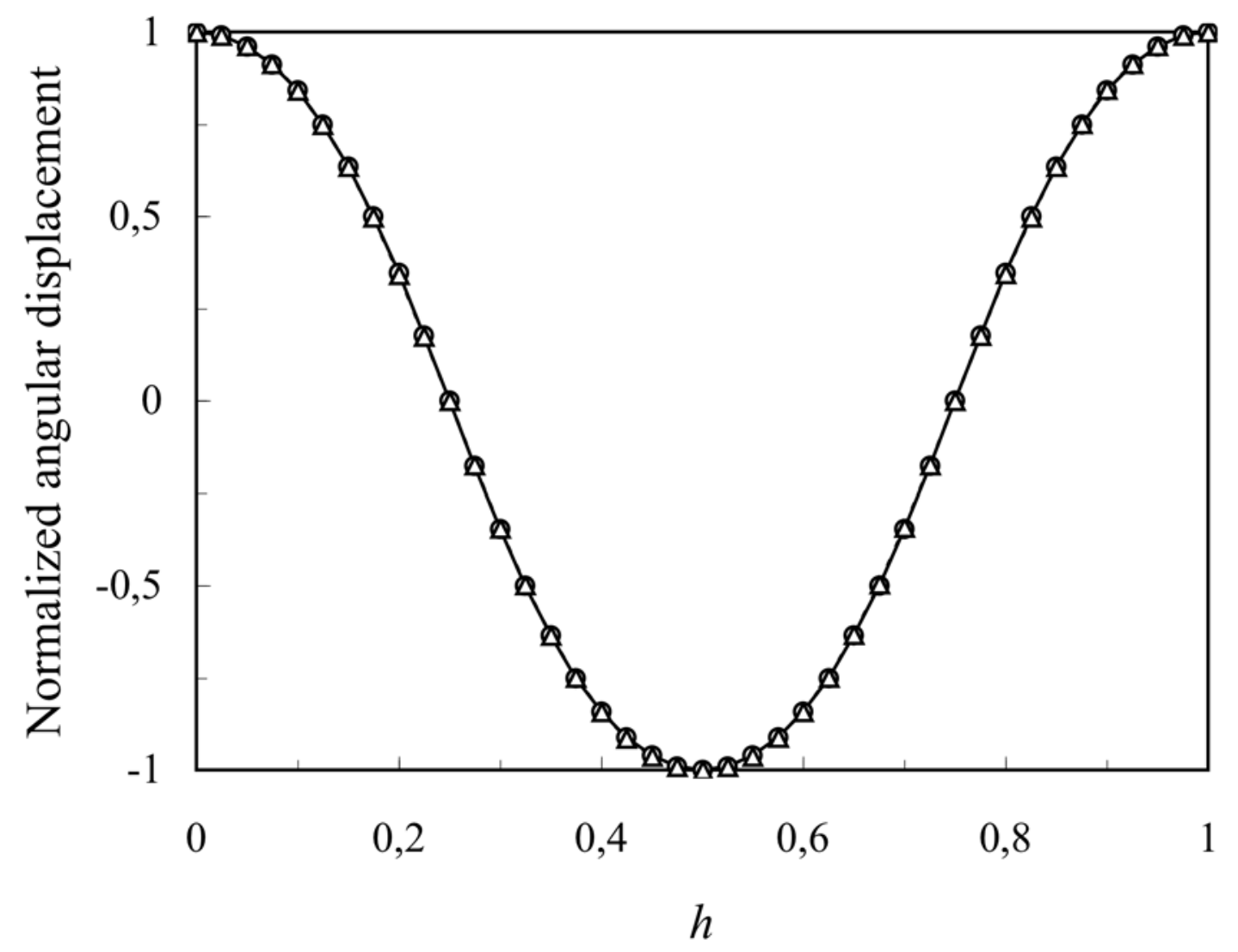

